\title{
Optimization of PBG-Waveguides for THz-Driven Electron Acceleration
}

\author{
Andrew Vint, Graeme Burt, and Rosa Letizia, Senior Member, IEEE
}

\begin{abstract}
The properties of two-dimensional photonic bandgap dielectric structures, also called photonic crystals, are numerically investigated to assist the design of waveguides for THz driven linear electron acceleration. Given the broadband nature of the driving pulses in $\mathrm{THz}$ acceleration regimes, one design aim is to maximise the photonic bandgap width to allow propagation of the relevant frequencies within the photonic crystal linear defect waveguide. The proposed design is optimized to provide the best compromise between effective acceleration bandwidth and strong beam-wave interaction at the synchronism central frequency. Considerations on achieved acceleration bandwidth, accelerating voltage and surface magnetic field are given to compare the proposed geometry to one of the main counterparts in the literature-the dielectric-lined waveguide.
\end{abstract}

Index Terms-terahertz electron acceleration, photonic crystals, photonic-bandgap waveguides

\section{INTRODUCTION}

W ITH current state-of-the-art particle accelerators reaching several kilometres in size, a paradigm shift is required for future technologies to prevent them becoming unattainable. Terahertz $(\mathrm{THz})$ frequencies offer the opportunity to build micron-scale structures, and the use of dielectrics over metal offers the ability to push fields higher due to the increased breakdown resistance-or fluence threshold. Additional benefits over optical frequencies, which similarly exhibit the prior stated benefits of $\mathrm{THz}$, is the larger aperture sizes allowed for the beam and higher bunch charges $\mathrm{THz}$ affords beams to use; as well as improved bunch quality due to a single cycle being able to capture ps-length bunches, allowing for monoenergetic beams [1].

In conventional RF-driven accelerators, sources are typically narrowband, usually on the order of less than a few percent. This allows the analysis to be performed around a central frequency assuming negligible phase-velocity slippage compared to the particle-beam being accelerated; thus can be assumed the whole bandwidth will contribute positively towards the acceleration voltage. In the regime of ultrashort $\mathrm{THz}$ pulse, broad bandwidths are generated and require a more careful study of the beam-pulse interaction due to the presence of frequencies which are far from phase-velocity synchronism. Thus the accelerating voltage becomes dependent on several factors such as: the length of the waveguide; the dispersion

Manuscript received Month, xx, 20xx. This work was supported by the Cockcroft Studentship grant ST/N504129/1.

A. Vint, G. Burt and R. Letizia are with the Lancaster University Department of Engineering: Lancaster University, Gillow Ave, Bailrigg, Lancaster, LA1 4YW; and the Cockcroft Institute, Keckwick Lane, Daresbury, Warrington WA4 4AD (email: andrew.vint@cockcroft.ac.uk; g.burt1@lancaster.ac.uk; r.letizia@lancaster.ac.uk).

Digital Object Identifier NUMBER of the accelerating mode in the waveguide; and the spectral interaction impedance across all frequencies propagating.

One current limiting factor in high-gradient devices is field magnitude on the accelerating structure walls: high electric fields leading to surface breakdown and field emission; and high magnetic fields which can cause pulsed heating leading to surface deformation and degradation [2], [3]. Linear acceleration of electrons at this frequency range needs to take into account the aforementioned aspects. Dielectric lined waveguides (DLW), both rectangular and cylindrical, have been proposed in the literature [4]. Due to the wavelength scales of $\mathrm{THz}$, employing photonic crystal $(\mathrm{PhC})$ technology for lateral electromagnetic confinement instead of metal walls can offer lower ohmic losses [5], [6], potential for damping higher-order modes, and high fluence threshold. In particular, as the fields evanescently decay away from the vacuum channel, they are of negligible amplitude by the point any metal supports or structure are encountered. In this paper, the use of a photonic crystal waveguide, periodic in two dimensions, is proposed to replace the dielectric lined metal wall of a DLW type structure to minimize wall fields.

The design flexibility offered by $\mathrm{PhCs}$ is investigated to tailor the accelerating mode dispersion for the $\mathrm{THz}$ acceleration regime while simultaneously allowing for machining tolerances $>5 \%$ [7]. For comparison with other designs such as the DLW proposed in [8] and ease of realisation, a rectangular waveguide configuration is chosen. This work focuses on waveguides based on a 2D-PBG slab structure with a triangular lattice of air/vacuum holes, offering a good compromise between design flexibility and ease-of-manufacture. The investigation carried out here focuses on accelerating voltage, acceleration bandwidth, and field intensity on the metal/vacuum interface in the channel obtainable from a 100 $\mathrm{mJ}$ pulse. While $100 \mathrm{~mJ}$ is large, it is not unrealistic to expect pulses of this magnitude being available in the next few years [9].

\section{BROADBAND THz Pulses}

For lasers to achieve high powers at $\mathrm{THz}$ frequencies, it is required that the pulse is short, typically on the order of picoseconds. This has the consequence that the Fourier transform of the pulse has a broadband energy spectral distribution (ESD). Narrowband ESD pulses can be approximated as a pure gaussian distribution:

$$
\widetilde{U}(\omega)=U_{\max } \exp \left[-\frac{\left(\omega-\omega_{0}\right)^{2}}{2 \sigma^{2}}\right](J / H z)
$$


where $\omega_{0}$ is the centre frequency, $E_{\max }$ is the spectral energy at the centre-frequency, and $\sigma$ is the standard deviation of the curve. Broadband ESD pulses can however show a skew in the distribution. This skew can be expressed as a modified Gaussian distribution, shown in Eqn. 2.

$$
\widetilde{U}(\omega)=U_{\max } \exp \left[-\left(\frac{\left(\omega-\omega_{0}\right)}{\sqrt{2} \sigma}\right)^{2}\left(\frac{\omega_{0}}{\omega}\right)^{x}\right](J / H z)
$$

where $x$ is the source-dependent frequency-decay coefficient. Thus equations 1 and 2 are consistent when $x=0$. This gives the expected exponential decay profile at frequencies higher than $\omega_{0}$ while preserving the Gaussian distribution below $\omega_{0}$. This can be seen in Fig. 1.

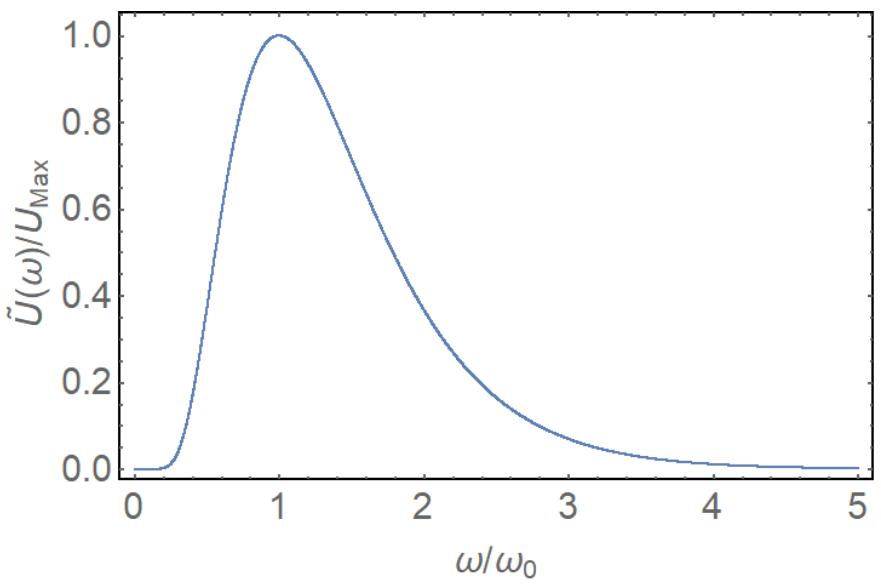

Fig. 1. Spectral energy distribution curve for broadband $\sigma=0.5 \omega_{0} \mathrm{THz}$, $x=1$ pulse, normalised for clarity.

The pulse which was used for analysis was $\omega_{0}=1 \mathrm{THz}$ with $E_{\max }$ for each $\sigma$ calculated to provide $\int_{0}^{\infty} \widetilde{U}(\omega) d \omega=$ $100 \mathrm{~mJ}$. In addition, the frequency-decay coefficient is taken as $x=1$ for simplicity in analysis. To calculate the accelerating bandwidth, the spectral voltage profile for the accelerating mode is required. This was obtained via:

$$
\widetilde{V}(\omega)=\mathbb{R}[T(\omega)] \sqrt{\widetilde{U}(\omega) Z_{c}(\omega)}(V / H z)
$$

where $Z(\omega)$ is the spectral characteristic impedance and $T(\omega)$ is the spectral transit time factor, calculated by:

$$
T(\omega)=\frac{1}{L} \int_{0}^{L} \exp \left[i \omega z\left(\frac{1}{v_{p}(\omega)}-\frac{1}{v_{b}(\omega)}\right)\right] d z
$$

where $L$ is the length of the structure, $v_{p}(\omega)$ is the spectral phase velocity of the accelerating mode, and $v_{b}(\omega)$ is the spectral particle velocity. In a structure of length $L$, some asynchronous frequencies will propagate with a net accelerating effect, others will propagate with a net decelerating effect. Integrating over all frequencies as in Eqn. 5 gives the total accelerating voltage. Calculating Eqn. 2 for increasing $\sigma$ allowed Eqn. 5 to be plotted against $\sigma$ which illustrates the optimal source bandwidth for the structure acceleration in the next sections.

$$
V_{a c c}=\int_{0}^{\infty} \tilde{V}(\omega) d \omega(V)
$$

\section{PBG-WAVEGUIDE DESIGN}

\section{A. Dielectric Choice}

The PBG-W is designed to: sustain a fundamental TMlike mode with strong longitudinal E-field for acceleration of an electron beam; be single moded; have a photonicbandgap large enough to encompass the broadband $\mathrm{THz}$ pulse and optimise beam-wave interaction over a broad range of frequencies.

For a photonic-bandgap waveguide (PBG-W) to be useable in accelerator applications beyond proof-of-concept, it not only needs to perform well in relation to high-gradient acceleration characteristics but also must be manufacturable. Certain accelerating characteristics, such as those detailed in later sections, can be used as figures of merit (FoMs) for comparing structures without requiring full particle dynamic calculations to be undertaken. Pushing for the highest acceleration FoMs in photonic structures can lead to devices which are complex and, at the tens-of-microns scale required for $\mathrm{THz}$ structures, are not easy to manufacture. Moreover, complexity in small structures can be unsuitable to high power operation. PBG-Ws built using photonic crystals which are periodic in two dimensions offer a good compromise between acceleration FoMs and manufacturability. A 2D PBG-W was proposed in [10] for acceleration in the optical frequency range, which led to using a $2 \mathrm{D}$ photonic crystal of bulk high refractive index $n$ dielectric with low- $n$ cylindrical holes in a triangular spacing, as shown in inset in Fig. 2, perpendicular to the propagation axis of the particles. Confinement in the perpendicular direction, $\mathrm{x}$-axis, is achieved by truncating the slab thickness to a finite value and cladding it with two metal plates, as shown in Fig. 3.

The choice of dielectric dictated the rest of the structure's design. The photonic-bandgap width is proportional to the ratio of permittivities of the two constituent media of the $\mathrm{PhC}$ [11], thus the higher relative permittivity $\varepsilon_{r}$ for the dielectric surrounding the air holes, the broader the bandgap. Higher $\varepsilon_{r}$ however has the opposing effect of both increasing the manufacturing precision required near the channel due to smaller regions having greater effect on the field; and reducing the group velocity $v_{g}$ through the structure as defining the refractive index, which details the change in phase-velocity:

$$
n(\omega)=\sqrt{\varepsilon_{r}(\omega) \mu_{r}(\omega)}
$$

where the relative permeability $\mu_{r}=1$ for most dielectrics. As such:

$$
v_{p}(\omega)=\frac{c}{n(\omega)}(m / s)
$$

Which leads onto defining the group index, which details the corresponding change in group velocity in dielectric media:

$$
n_{g}(\omega)=\frac{c}{v_{g}(\omega)}=c\left(\frac{d \omega}{d k}\right)^{-1}=\frac{d}{d \omega}[\omega n(\omega)]
$$


Where $c$ is the speed of light. Thus applying the chain rule we get:

$$
n_{g}(\omega)=n(\omega)+\omega \frac{d n(\omega)}{d \omega}
$$

As illustrated later in Section III-D, the group velocity affects the overall acceleration bandwidth of the structure. A common material used in photonic structures for particle acceleration is silica $\left(\mathrm{SiO}_{2}\right)$ [8], which has $\varepsilon_{r} \approx 3.5$ at $\mathrm{THz}$ frequencies. While this allows for expected group velocities of $v_{g}=0.63 c$ at $v_{p} / c=1$, the maximum bandgap for the structure in this study was found to be approximately $16 \%$ which is not suitable to confine a broadband $\mathrm{THz}$ pulse. The dielectric material chosen was silicon, with higher relative permittivity $\varepsilon_{r} \approx 11.6$ and loss-tangent $\tan \delta<10^{-3}$ [12]. This material was chosen for investigation due to availability of data, mature production technology, and a value of $\varepsilon_{r}$ which would permit a PBG wide enough to encompass the majority of a broadband $\mathrm{THz}$ pulse.

\section{B. Waveguide Design}

As shown in [10], the choice of a triangular lattice of holes in high permittivity medium opens a bandgap for the longitudinal-TM polarization of the electromagnetic modes, where the E-field has components in the plane of the 2D structure. This allows the design of a PBG-W which can support a fundamental TM-like mode with a strong longitudinal Efield component suitable for acceleration. As a reference, the central frequency of $1 \mathrm{THz}$ is assumed. Using the software MPB [13], 2D simulations of the PhC unit cell were carried out to optimize the fill factor of the periodic structure for maximum PBG around the central frequency.

The maximum PBG was found at a hole radius of $r=0.445 a$ yielding a PBG of $50 \%$ in the normalized frequency region of $0.29-0.48 \omega a / 2 \pi c$, where $\omega$ is the angular frequency, $a$ is the PhC period, and $c$ is the speed of light. The band diagram for this case is shown in Fig. 2. Central frequency of $1 \mathrm{THz}$ is realized for period $a=117 \mu \mathrm{m}$.

The PBG-W was realized by introducing a line-defect along the longitudinal direction $z$ across the photonic-crystal lattice for confinement of the accelerating mode, with channel width of $w$, as shown in Fig. 3. The PBG slabs were then clad between metal plates at the $x$-axis boundaries. A solid dielectric lining placed between the bulk $\mathrm{PhC}$ and the vacuum channel, the pad layer, was added to offer an additional method of control over the accelerating mode dispersion in the waveguide. It was found that truncating the PBG slabs at the $y$-axis boundaries and the addition of metal boundaries did not significantly alter the band diagram shown in Fig. 2

\section{Characteristic Impedance}

Firstly, the channel width and pad layer thickness were investigated using $2 \mathrm{D}$ eigenmode simulations to obtain a suitable dispersion of the accelerating mode at the point of interaction with $v_{p}=c$ and $f=1 \mathrm{THz}$. This was found at channel width $3 a$ and pad-layer of $0.1 a$ providing a starting point for the design of the $3 \mathrm{D}$ waveguide and its optimization

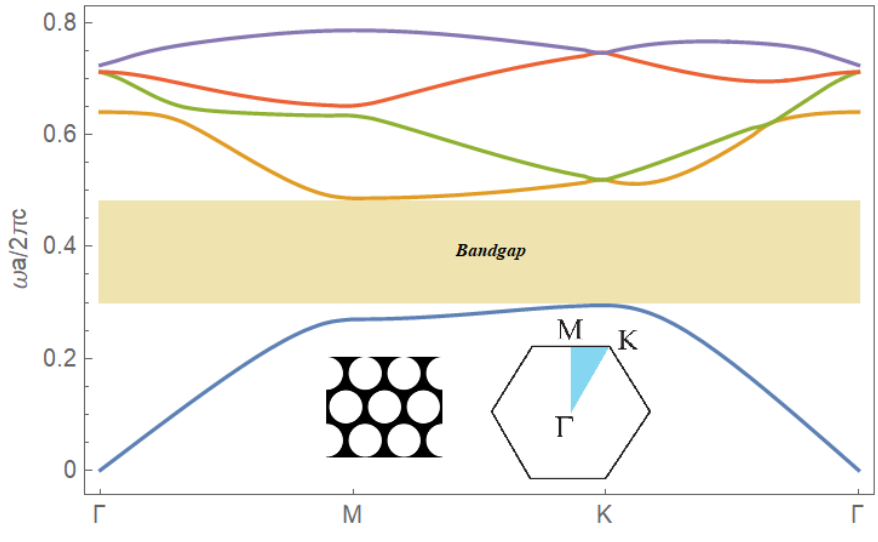

Fig. 2. Band diagram for the 2D-PBG structure of $r=0.445 a$ and $\varepsilon_{r}=$ 11.66 for $\mathrm{TM}$ polarization showing bandgap centered on $\omega a / 2 \pi c \sim 0.4$ corresponding to $1 \mathrm{THz}$ for $a=117 \mu \mathrm{m}$. The PBG structure-where black is dielectric and white is vacuum, and irreducible Brillouin zone are shown in inset to the left and right respectively.

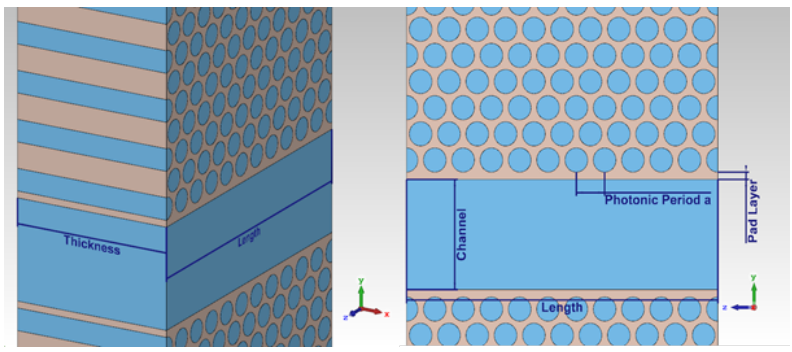

Fig. 3. Schematic diagram of the photonic-crystal waveguide-where blue is vacuum, and orange is dielectric.

for highly relativistic $\mathrm{THz}$ acceleration. One of the main FoMs in conventional RF accelerators is the shunt impedance $r_{s}$. This is defined, as in Eqn. 10 as the ratio of the accelerating longitudinal field $E_{a c c}$ to the power dissipated on the walls of the cavity $P_{\text {loss }}$ per unit length. [14]

$$
r_{s(\text { def })} \equiv \frac{E_{a c c}^{2}}{\frac{\delta P_{\text {loss }}}{\delta z}}
$$

Thus in a known length of cavity $L$, assuming the field is not varying longitudinally, Eqn. 10 becomes Eqn. 11:

$$
r_{s}=\frac{E_{a c c}^{2} L T}{P_{\text {loss }}}=\frac{(V T)^{2}}{P_{\text {loss }}}
$$

where $T$ is the transit-time factor, or the voltage seen by a particle under the influence of amplitude variation of the accelerating field; and $V$ is the accelerating voltage along the structure. This FoM is typically not used for PBG structures for accelerator applications due to the wall-losses being a small component of the total power figure in the structure. The characteristic impedance $Z_{c}$ is used in the context of dielectric laser accelerators. This is defined as the product of the accelerating field $E_{a c c}$ with the integrated Poynting vector $S$ giving average power flow in the direction of acceleration in the structure [15] via Eqn. 12: 


$$
Z_{c(\text { def })} \equiv \frac{E^{2}}{\int S \delta A}=\frac{E^{2}}{P_{\text {flow }}} \quad\left(\Omega / m^{2}\right)
$$

Thus to ensure Eqn. 12 has the correct units of $\Omega$, the value is calculated for a structure of one accelerating wavelength $\lambda$ long which allows scalability between structures designed for different wavelengths. With this, Eqn. 12 becomes Eqn. 13:

$$
Z_{c}=\frac{E^{2} \lambda^{2}}{P_{\text {flow }}}=\frac{V_{\lambda}^{2}}{P_{\text {flow }}}
$$

where $V_{\lambda}$ is the accelerating voltage per wavelength in the structure. Due to the structure being dispersive, $\lambda$ is not constant with $\omega$ and is extracted from the wave-vector $k_{z}$ as $\lambda(\omega)$. When $Z_{c}$ for one wavelength is known, $Z_{c}$ for structures of length $L$ can be calculated via:

$$
Z_{c}=\frac{V_{\lambda}^{2}}{P_{\text {flow }}} \frac{L}{\lambda(\omega)}
$$

It was found that the accelerating mode tends infinitely to zero at the metal walls, so lower losses were expected than in a metal waveguide structure. The thickness of the PBG-W slab and the channel width were investigated to study optimization of the structure-particle beam interaction for broadband pulse excitation by comparing characteristic impedance [15]. While $Z_{c}$ is often used in literature, a more detailed analysis was required to incorporate the broadband nature of the excitation pulse.

The CST eigenmode solver [16], was used to investigate the effects of channel width on the PBG-W at the point of beamwave interaction in structures with $a=117 \mu m$, hole radius $r=0.445 a$, and pad layer of $13 \mu \mathrm{m} . Z_{c}$ was calculated for PBG-Ws ranging in thickness from $283-425 \mu \mathrm{m}$ and channel width $266-398 \mu \mathrm{m}$. In performing the calculations, the structures were extremely sensitive to mesh imperfections. As such, the mesh was set to $5 \lambda^{-1}$ with 6-pass mesh refinement for $<0.1 \%$ frequency error, leading to approximately $1.5 \%$ error in parameter calculations.

\section{Dependence on Group Velocity}

For the structure investigated at the end of the previous section, the group velocity ranged between $v_{g} \approx 0.18 c-0.23 c$. To determine how this will affect the accelerating voltage in the structure, another FoM was required. Combining $Z_{c}$ with the group velocity as in Eqn. 15 provided a more in-depth examination of the acceleration properties of the structure and quantifies the accelerating voltage produced from a stored energy.

$$
\frac{R}{Q}=\frac{Z_{c} v_{g}}{2 \pi \lambda c} \quad(\Omega / m)
$$

Thus calculating $R / Q$, the ideal waveguide dimensions for this structure were in the region of the channel width $300-340 \mu m$ and waveguide thickness $360-420 \mu m-$ giving a $R / Q$ between $12 k \Omega / m-15 k \Omega / m$.

Similarly to $Z_{c}$, the R/Q is an adequate FoM for analysis of single-frequency excitation structures. The final accelerating voltage of a structure excited by a broad bandwidth pulse however depends on both $R / Q$, and the synchronism of the pulse through the structure: both pulse-envelope and phaseangle synchronism as to maximize the transit-time factor. Examining the process of calculating the voltage in Section II, the calculation for the accelerating voltage in Eqn. 3 and Eqn. 5 has several proportionality factors beyond simply increasing the accelerating electric field. From this it can be inferred the voltage is proportional to the group velocity:

$$
T(\omega) \propto v_{g}
$$

Thus from Eqns. 3 and 16 it can be seen:

$$
V(\omega) \propto v_{g} \sqrt{Z_{c}}
$$

Also from Eqn. 15 we can see that:

$$
\frac{R}{Q} \propto v_{g} Z_{c}
$$

Thus:

$$
V \propto \sqrt{v_{g} \frac{R}{Q}}
$$

Therefore from this a new figure of merit was introduced, the velocity-modified R/Q coefficient $\zeta_{v}$ :

$$
\zeta_{v}=v_{g} \frac{R}{Q}
$$

It is important to note this derivation does not incorporate higher-order effects such as group-velocity dispersion or thirdorder dispersion, therefore is an approximation. Maximizing this factor in structures at the interaction point provides a design starting point for pursuing the maximum accelerating voltage across the total frequency bandwidth.

\section{E. Dispersion and Accelerating Bandwidth}

Numerical simulations showed that the $\zeta_{v}$ is maximized for $a=82.201 \mu \mathrm{m}$ and $r=28.7 \mu \mathrm{m}$ when channel width is taken as $330 \mu \mathrm{m}$ and thickness is $350 \mu \mathrm{m}$. The profile of the accelerating mode and its dispersion are shown in Fig 4 and Fig. 5, respectively.

It can be seen from Fig. 5 that there is only one frequency which is synchronous with $v_{b}=c$ particles, thus for narrowbandwidth applications further frequency-domain calculations could yield total accelerating voltage from this alone. As the focus of this paper is broadband acceleration, the analysis reported in Section II is applied for a waveguide of length $L=5 \mathrm{~mm}$. This gives a total acceleration voltage as seen in Fig. 6 showing a maximum acceleration voltage of $\approx 1.1 \mathrm{MV}$ at a relative bandwidth of $\sigma \approx 1 \%$ before falling rapidly. As can be seen in Fig. 5, the point of interaction is high on the curve and at a point with low gradient. As a result, there is only a narrow region in which the phase-synchronism condition is met over the length of the structure. This leads to the poor accelerating voltage distribution shown in Fig. 6 which does not allow effective acceleration for broadband driving pulses. 


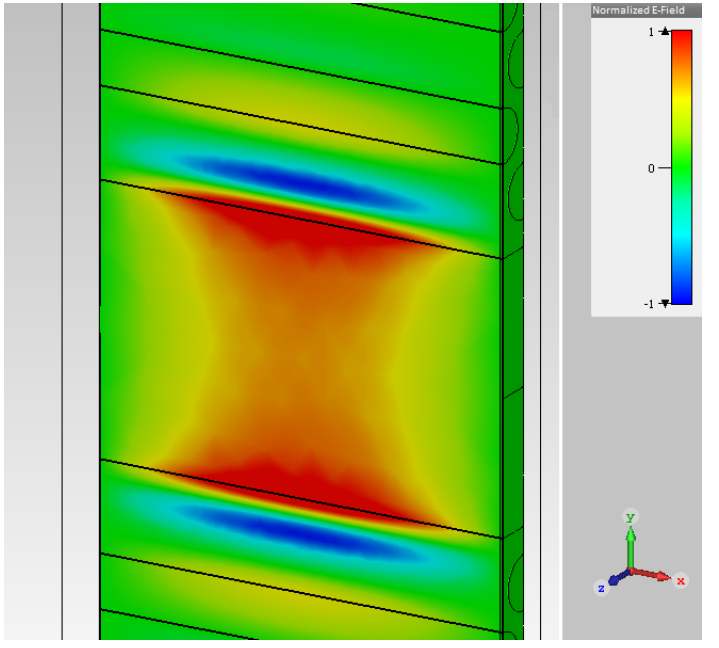

Fig. 4. Normalized $E_{z}$ component of the accelerating mode in PBG-W, the cylinders of the $\mathrm{PhC}$ structure visible top and bottom.

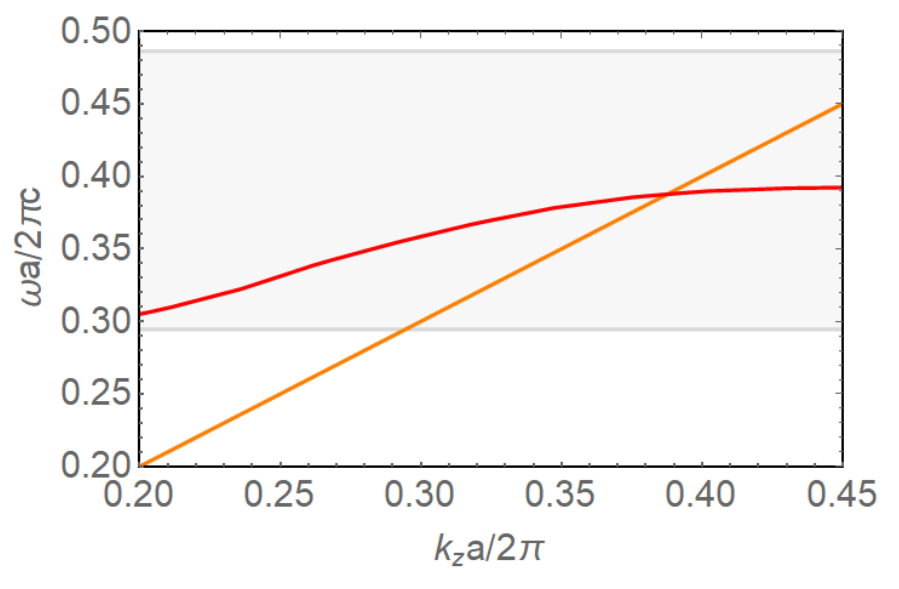

Fig. 5. Accelerating mode dispersion for the PBG-W, where $a$ is the photonic crystal period, with superimposed speed-of-light (SoL) line. Synchronism between particle velocity and phase velocity of the accelerating mode is realized at the intersection of these two curves.

\section{F. Field Distribution Ratio}

One of the main reasons to pursue PBG-W structures is the ability to reduce the E-field on the metal walls compared to metal-based structures such as the DLW. Whilst previous FoMs address the particle accelerating capability of the PBGWs, additional figures were required to address the field characteristics. These are:

$$
\eta_{W a l l}=\frac{E_{W a l l}}{E_{A c c}}
$$

and:

$$
\eta_{M a x}=\frac{E_{M a x}}{E_{A c c}}
$$

where $E_{W \text { all }}$ is the maximum electric field found on the metal wall of the structure, $E_{A c c}$ is the maximum accelerating gradient, and $E_{M a x}$ is the maximum electric field found in the

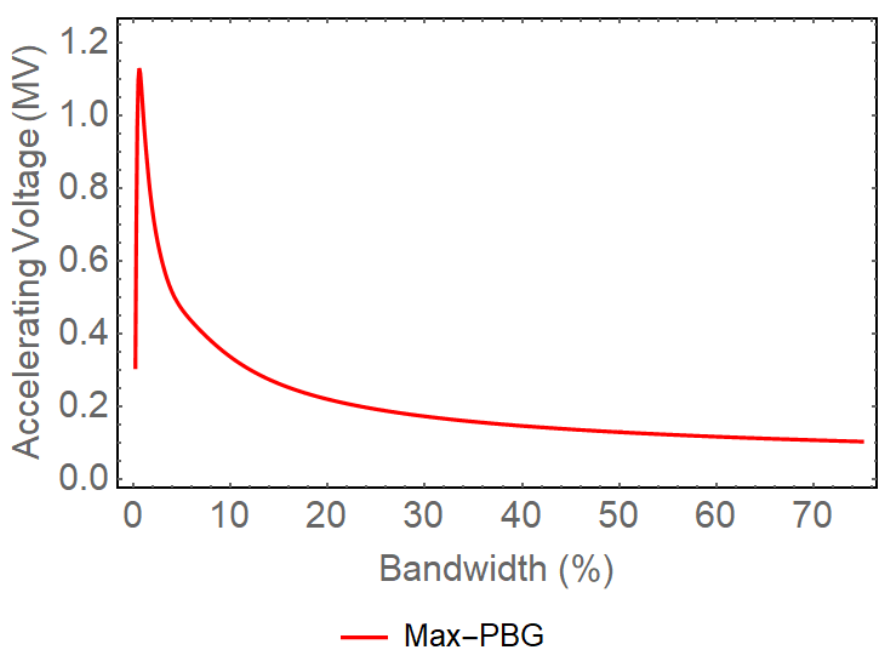

Fig. 6. Accelerating voltage variation with driving pulse bandwidth obtained for $r=0.445 a$ (maximum PBG width).

structure. For the structures analyzed in this paper, $\eta_{W \text { all }}$ will examine the maximum field on the metal/vacuum interface in the channel of the waveguide, as this is the region where minimizing fields which could contribute to field-emission would be beneficial.

For the structure which generated the accelerating voltage in Fig $6, \eta_{W \text { all }}=4.24$ and $\eta_{\text {Max }}=14.29$ showing despite a broad bandgap, this structure has poor electromagnetic confinement for beam interaction. As such, it can be inferred that the bulk of the accelerating field resides in the dielectric and does not contribute towards acceleration.

\section{Optimizing the PBG-W FOR THz Pulses}

Optimization of the PBG-W for $\mathrm{THz}$ driven acceleration aims at finding a compromise between accelerating voltage and effective acceleration bandwidth. Broader coupling of the driving field with the particles can be realized if the interaction is moved further down the dispersion curve into the higher gradient region, leading to lower $v_{g}$ dispersion. To achieve this, the bandgap center-frequency must be reduced so that the bandgap encompasses a lower wavenumber region of the SoL line. For this lattice geometry, it can be seen a decrease in center-frequency will also result in a reduced PBG width [11]. As such a point must be selected which is an acceptable compromise between both PBG-width and center-frequency. Selecting a hole radius $r=0.350 a$ led to a photonic-bandgap of $41 \%$ in the region $0.22-0.33 \omega a / 2 \pi c$ which still encompassed the accelerating mode around the frequencies of interest. The band diagram for this structure can be seen in Fig. 7.

Performing the analysis as in Section III-D, the $\zeta_{v}$ variation with channel-width and thickness in this PBG-W with hole radius $r=0.350 a$ is shown in Fig. 8. As can be seen, the relationship between channel-width and thickness is not linear. A general trend can however be seen as narrower channel, and a thicker slab yields higher $\zeta_{v}$. A point was chosen at channelwidth $300 \mu \mathrm{m}$ and thickness $470 \mu \mathrm{m}$ for further investigation. For manufacturability, this structure also had the photonic 


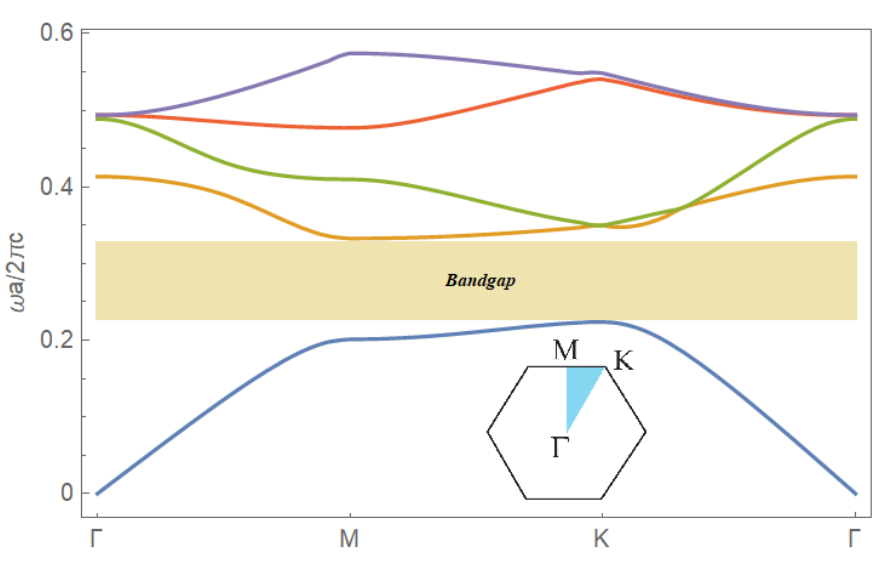

Fig. 7. Band diagram for the 2D-PBG structure of $r=0.350 a$ and $\varepsilon_{r}=$ 11.66 for TM polarization showing bandgap centered on $\omega a / 2 \pi c \sim 0.28$. The irreducible Brillouin zone is shown in inset.

period $a$ modified from $a=82.201 \mu \mathrm{m}$ to $80 \mu \mathrm{m}$, the hole radius modified from $r=0.350 a(28.7 \mu \mathrm{m})$ to $r=30 \mu \mathrm{m}$, and the pad layer was set to $30 \mu \mathrm{m}$. This reduced the $\zeta_{v}$ from $\zeta_{v} \approx 4 \times 10^{12}$ to $\zeta_{v}=2.45 \times 10^{12}$.

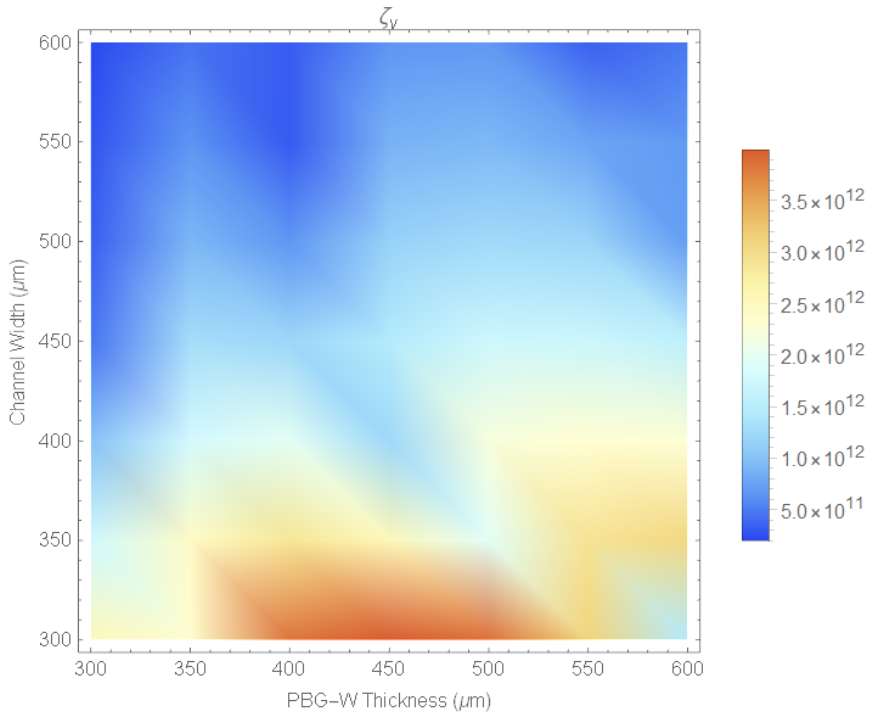

Fig. 8. $\zeta_{v}$ variation with PBG-W thickness and channel width for structure $r=0.350 a$.

For comparison with the high $\zeta_{v}$ case, a further structure maximizing $v_{g}$ was identified at $600 \mu \mathrm{m}$ channel, $900 \mu \mathrm{m}$ thickness and a $20 \mu \mathrm{m}$ pad layer. This structure had a considerably lower spectral $Z_{c}$ but had a phase-synchronous group velocity of $v_{g} \approx 0.71 c$ and thus provided a good comparison for the effects of both $\zeta_{v}$ and $v_{g}$ on the accelerating voltage. Obtaining the dispersion and spectral $Z_{c}$ for both, as shown in Fig. 9, the accelerating voltage bandwidth was calculated and plotted in Fig. 10. It can be noted that the acceleration bandwidth showed improvement in correlation with $v_{g}$. For the high- $\zeta_{v}$ case, $\eta_{W \text { all }}=0.69$ and $\eta_{M a x}=3.99$; showing improvement over the max-PBG structure. On the other hand, the high group velocity structure has a $\eta_{W \text { all }}=0.45$ and $\eta_{\text {Max }}=9.28$ showing that while synchronism is good, much

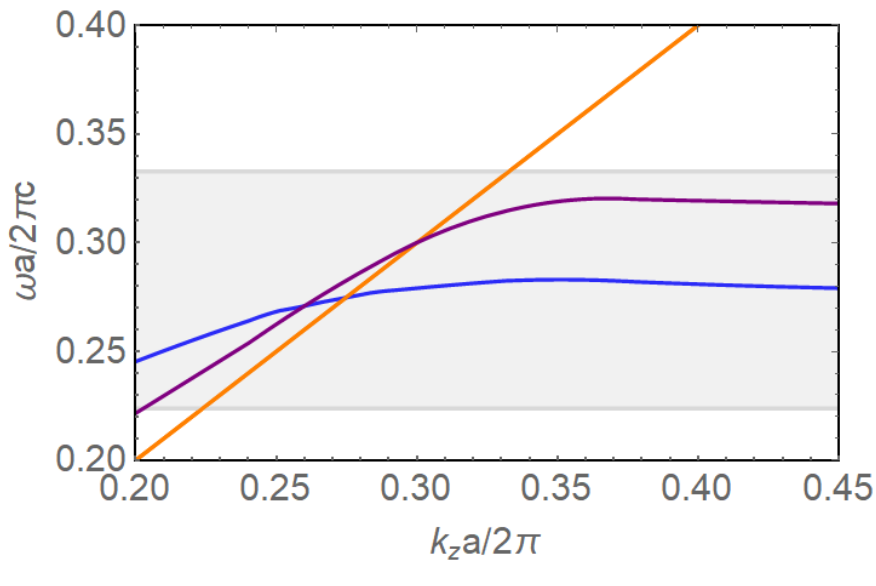

$-\mathrm{High}-\zeta_{v}-\mathrm{High}-v_{g}-$ SoL - Bandgap

Fig. 9. Dispersion curves for two structures identified with $r=0.350 a$, one optimized for high- $\mathrm{R} \zeta_{v}$, and one optimized for high- $v_{g}$.

of the field is being wasted.

Optimization of the proposed PBG-W for broad bandwidths for the high-R/Q structure improved the resultant $25 \%$ bandwidth acceleration voltage from $\sim 0.25 \mathrm{MV}$ to $\sim 0.6 \mathrm{MV}$, with a peak acceleration voltage of $\sim 1.1 \mathrm{MV}$ available, while doubling the accelerating voltages at larger bandwidths. For the high group-velocity structure, the $25 \%$ bandwidth acceleration voltage was further increased to $\sim 0.8 \mathrm{MV}$ with improved acceleration voltage over the high $\zeta_{v}$ structure at higher bandwidths, with the cost of much poorer acceleration voltages at low bandwidths. This is expected to be due to a component of the electric field propagating into the dielectric and thus not contributing to acceleration.

While this structure was made from silicon, first-order analysis of Eqn. 9 shows the expected group velocity of a dielectric-coupled structure is $v_{g}=0.29 c$ which will poorly affect both bandwidth and $\zeta_{v}$. To achieve a group velocity of $v_{g}>0.5 c$ would be more desirable and as such a material with $\varepsilon_{r}<4$ would be required. The use of silica was discounted as the maximum bandgap of a structure using this material was investigated and found to be approximately $16 \%$ and as such too low for adequate broadband acceleration. Alternatively, materials such as titanates of barium or strontium with an extremely high permittivity could be worth investigating.

\section{COMPARISON WITH DLW}

To fully assess the capabilities of the proposed PBG waveguide, a comparison with the dielectric-loaded waveguide (DLW), whose properties were investigated for $\mathrm{THz}$ driven acceleration in [8], is performed.

The structure presented in [8] was rescaled for an interaction frequency of $1 \mathrm{THz}$, and the dielectric was changed from quartz to silicon for direct comparison-which involved changing the thickness of the dielectric layer to compensate for the increase in refractive index. The geometry for all structures examined is given in Table I. The accelerating mode dispersion found in the DLW is shown in figure 11. Figure 12 compares the accelerating voltage for all the structures investigated. 


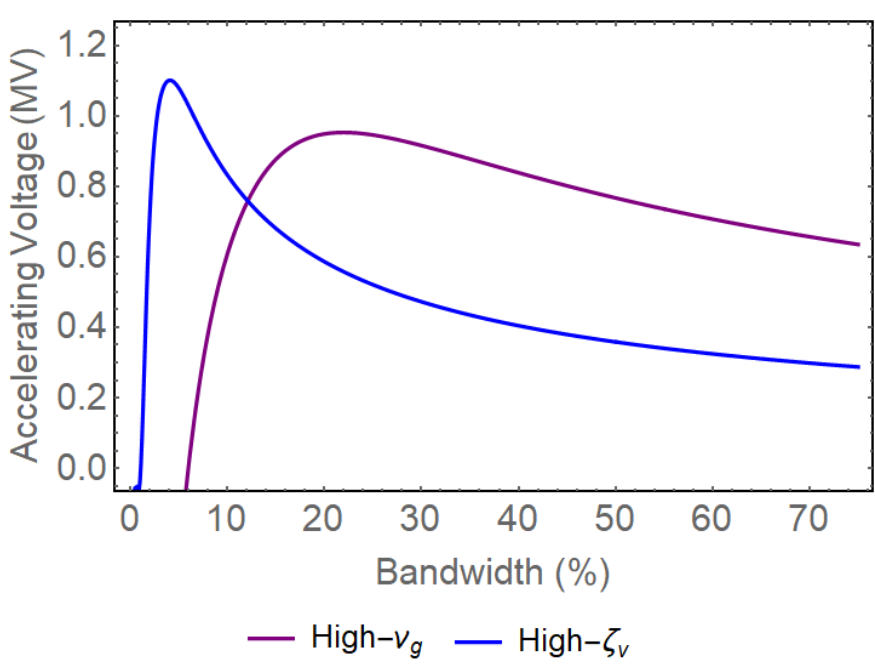

Fig. 10. Comparison of broadband accelerating voltage obtained in high- $\zeta_{v}$ and high- $v_{g}$ structures.

From Fig. 12 it can be seen that a large discrepancy in accelerating voltages exists between PBG-Ws and the DLW for the same input pulse. In comparison, the DLW had a $\zeta_{v}=1.43 \times 10^{13}$, a 5.83 -times increase over the high $\zeta_{v}$ PBGW structure. From Fig. 11 it can be seen that the dispersion for the DLW crosses the SoL line with higher $v_{g}$ and diverges less from it than any PBG-Ws examined. This led to a larger frequency-spectrum in which the phases move synchronously with the beam in $T(\omega)$ thus leading to a larger accelerating bandwidth for the structure. In regards to field distribution, the ratios for the DLW are $\eta_{W \text { all }}=0.31$ and $\eta_{\text {Max }}=3.06$, showing less field propagates within the dielectric layers, thus improving field contribution towards acceleration.

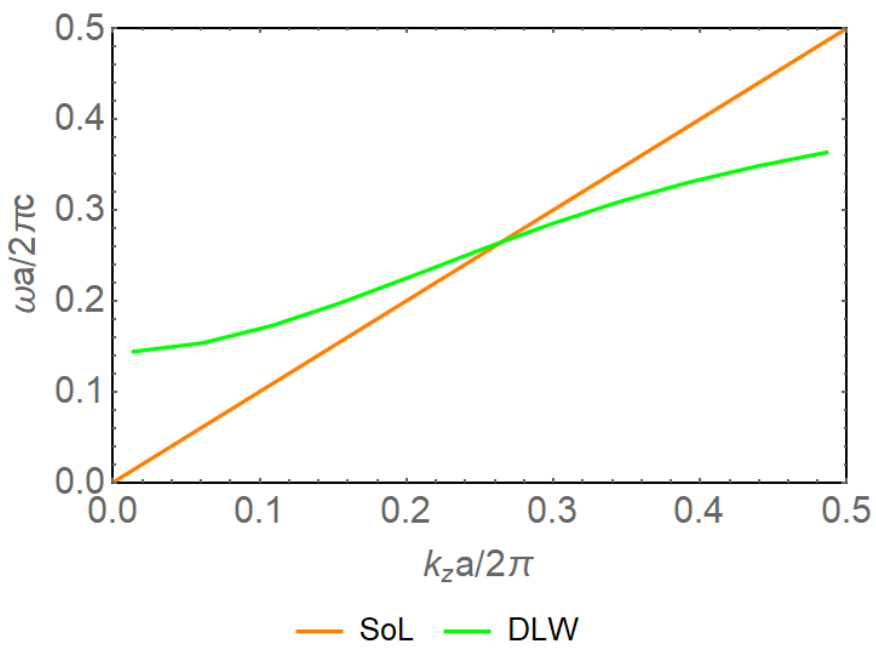

Fig. 11. Accelerating mode dispersion in silicon-loaded DLW, where $a$ is the length of the unit-cell simulated.

The PBG-W however provides a significant reduction of incident magnetic field on the wall compared to the DLW. Similar to the E-field distribution ratios, the magnetic ratio was used, where $\eta_{H}=H_{W \text { all }} / E_{A c c}$. Examination of the figures of merit for the structures under investigation in Table

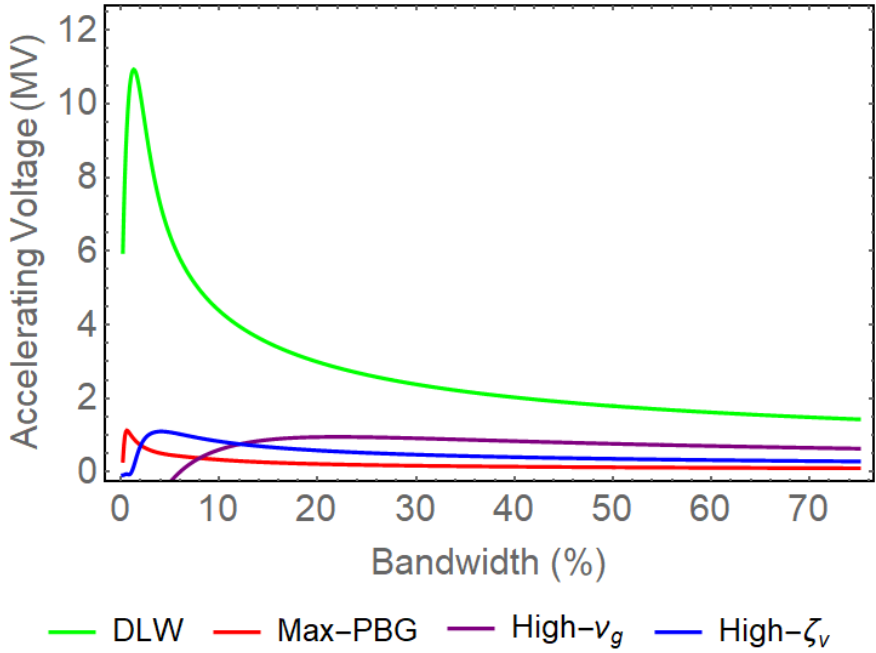

Fig. 12. Comparison of accelerating voltage for broadband pulses in all structures discussed.

I reveals that even poorly-optimized PGB-Ws have lower peak magnetic field on the wall, with a reduction of over $57 \%$ for the same accelerating voltage. While these fields do not have an effect on acceleration, their reduction will lead to lower heating effects. This can be seen to have been captured in the shunt impedance figures, with the optimized PBG-W having a $44 \%$ higher shunt impedance for the same pulse. As such, while the PBG-Ws investigated have not reduced peak electric fields on the metal walls of the vacuum channel compared to the DLW, total field incident on the wall leading to resistive losses has been reduced.

TABLE I

COMPARISON OF FIGURES FOR THE INVESTIGATED STRUCTURES.

\begin{tabular}{|c|cccc|c|}
\hline FoM & Max-PBG & High $-\zeta_{v}$ & High- $v_{g}$ & DLW & Unit \\
\hline Channel & 332 & 300 & 600 & 543 & $\mu m$ \\
Thickness & 354 & 470 & 900 & 600 & $\mu m$ \\
Radius & 47 & 30 & 30 & N/A & $\mu m$ \\
Pad Layer & 22 & 30 & 7 & 28 & $\mu m$ \\
\hline$Z_{c}$ & 27.84 & 162.96 & 27.84 & 244.79 & $\Omega / \lambda$ \\
$r_{s}$ & 3.71 & 75.26 & 24.89 & 52.15 & $M \Omega / m$ \\
$R / Q$ & 2.69 & 27.05 & 3.33 & 78.71 & $\mu \Omega / m$ \\
$v_{g} c$ & 0.18 & 0.30 & 0.66 & 0.61 & None \\
$\eta_{W \text { all }}$ & 4.24 & 0.69 & 0.45 & 0.31 & None \\
$\eta_{\text {Max }}$ & 14.29 & 3.99 & 9.28 & 3.06 & None \\
$\eta_{H}$ & 0.004 & 0.006 & 0.006 & 0.014 & N/A \\
\hline
\end{tabular}

\section{CONCLUSION}

The design of a photonic-crystal based waveguide to accelerate highly-relativistic electrons driven by $\mathrm{THz}$ radiation has been investigated. It was shown that to optimize the acceleration voltage from broad-bandwidth $\mathrm{THz}$ pulses, careful consideration needs to be paid towards photonic-bandgap width, accelerating mode dispersion, point of synchronism with the beam, and dielectric material. Results showed that although lower accelerating voltages overall were achieved compared to the DLW, the PBG-W here proposed can offer a suitable design for $\mathrm{THz}$ acceleration; outperforming the DLW in terms of magnetic fields on the walls and resistive losses. 
Important design considerations on the parameters governing optimization of the waveguide have been given.

\section{REFERENCES}

[1] E. A. Nanni, W. R. Huang, K.-H. Hong, K. Ravi, A. Fallahi, G. Moriena, R. D. Miller, and F. X. Kärtner, "Terahertz-driven linear electron acceleration," Nature communications, vol. 6, p. 8486, 2015.

[2] N. S. Ginzburg, I. I. Golubev, A. K. Kaminsky, A. P. Kozlov, S. V. Kuzikov, E. A. Perelstein, N. Y. Peskov, M. I. Petelin, S. N. Sedykh, A. P. Sergeev, A. S. Sergeev, A. A. Vikharev, and N. I. Zaitsev, "Experiment on pulse heating and surface degradation of a copper cavity powered by powerful $30 \mathrm{ghz}$ free electron maser," Phys. Rev. ST Accel. Beams, vol. 14, p. 041002, Apr 2011. [Online]. Available: https://link.aps.org/doi/10.1103/PhysRevSTAB.14.041002

[3] L. Laurent, S. Tantawi, V. Dolgashev, C. Nantista, Y. Higashi, M. Aicheler, S. Heikkinen, and W. Wuensch, "Experimental study of rf pulsed heating," Phys. Rev. ST Accel. Beams, vol. 14, p. 041001, Apr 2011. [Online]. Available: https://link.aps.org/doi/10.1103/PhysRevSTAB.14.041001

[4] S. Atakaramians, S. Afshar, T. M. Monro, and D. Abbott, "Terahertz dielectric waveguides," Advances in Optics and Photonics, vol. 5, no. 2, pp. 169-215, 2013

[5] P. Tassin, T. Koschny, M. Kafesaki, and C. M. Soukoulis, "A comparison of graphene, superconductors and metals as conductors for metamaterials and plasmonics," Nature Photonics, vol. 6, pp. 259-264, 2012.

[6] M. P. Kirley and J. H. Booske, "Terahertz conductivity of copper surfaces," IEEE Transactions on Terahertz Science and Technology, vol. 5, pp. 1012-1020, 2015.

[7] C. Matthews and R. Seviour, "Effects of disorder on the frequency and field of photonic-crystal cavity resonators," Applied Physics B, vol. 94, pp. 381-388, 2008.

[8] A. Healy, G. Burt, and S. Jamison, "Electron-terahertz interaction in dielectric-lined waveguide structures for electron manipulation," Nuclear Instruments and Methods A, vol. 909, pp. 199-203, 2018.

[9] C. Vicario, M. Jazbinsek, A. Ovchinnikov, O. Chefonov, S. Ashitkov, M. Agranat, and C. Hauri, "High efficiency thz generation in dstms, dast and oh1 pumped by cr: forsterite laser," Optics express, vol. 23, no. 4, pp. 4573-4580, 2015.

[10] B. M. Cowan, "Photonic crystal laser-driven accelerator structures," Ph.D. dissertation, SLAC-R-877, Stanford Linear Accelerator Center, 2575 Sand Hill Road, Menlo Park, CA 94025, 2007.

[11] J. Joannopoulos, S. Johnson, J. Winn, and R. Meade, Photonic Crystals, 2nd ed. Princeton University Press, 2008.

[12] P. Bolivar, M. Brucherseifer, J. Rivas, R. Gonzalo, I. Ederra, A. Reynolds, ..., and P. Maagt, "Measurement of the Dielectric Constant and Loss Tangent of High Dielectric-Constant Materials at Terahertz Frequencies," IEEE TRANSACTIONS ON MICROWAVE THEORY AND TECHNIQUES, vol. 51, pp. 1062-1066, 2003.

[13] "MIT Photonic Bands," mpb.readthedocs.io/en/latest/, accessed: 201901-22.

[14] T. P. Wangler, RF Linear Accelerators, 2nd ed. Wiley-VCH, 2008.

[15] R. England, N. R.J., K. Bane, D. Dowell, C. Ng, J. Spencer, ..., and R. Yoder, "Dielectric laser accelerators," Reviews of Modern Physics, vol. 86, pp. 1337-1389, 2014.

[16] "CST Microwave Studio, from Dassault Systemes," www.3ds.com/products-services/simulia/products/cst-studio-suite/, accessed: 2019-11-14.

[17] G. C. Burt, "Sweep-frequency microwave pulse compression using a helically corrugated waveguide," Ph.D. dissertation, Dept. Phys. University of Strathclyde, 16 Richmond Street, Glasgow, G1 1XQ, 2004.

[18] R. E. Collin, Field Theory of Guided Waves, 2nd ed. Wiley-Interscience, 1990.

[19] P. Lorrain, D. P. Corson, and F. Lorrain, Electromagnetic Fields and Waves, 3rd ed. W.H. Freeman and Company, 1987.

Andrew Vint was born in Scotland in 1987. He received, from the University of Strathclyde, Glasgow, UK, the MSci in Physics in 2010, and the MSc in High-Power Radio-Frequency Science and Engineering in 2011. Since 2016 he has been with Lancaster University at the Cockcroft Institute where he has been working on his Ph.D. on using Photonic Crystals for THz particle acceleration.
Graeme Burt is a professor in electronic and electrical engineering at Lancaster University and The Cockcroft Institute of Accelerator Science and Technology. His research interests are RF and $\mathrm{THz}$ structures for charged particle acceleration.

Rosa Letizia (M'13-SM'18) received the Laurea degree in electronic engineering from the Polytechnic of Bari, Bari, Italy, and the Ph.D. degree in computational photonics from the University of Leeds, Leeds, U.K., in 2005 and 2009, respectively. In 2011, she joined the Engineering Department, Lancaster University, Lancaster, U.K., and also the Cockcroft Institute, Warrington, U.K where she has been a Senior Lecturer since 2019. 